\title{
Narrow-line Seyfert 1's, water masers, and the peculiar case of IGR J16385-2057
}

\author{
Andrea Tarchi* \\ INAF-Osservatorio Astronomico di Cagliari, Loc. Poggio dei Pini, Strada 54, I-09012 \\ Capoterra (CA), Italy \\ E-mail: atarchi@oa-cagliari.inaf.it
}

\section{Paola Castangia}

INAF-Osservatorio Astronomico di Cagliari, Loc. Poggio dei Pini, Strada 54, I-09012

Capoterra (CA), Italy

E-mail: pcastang@oa-cagliari.inaf.it

\section{Francesca Panessa}

IASF/INAF, via del Fosso del Cavaliere 100, 00133 Roma, Italy

E-mail: francesca.panessadiasf-roma.inaf.it

\section{James A. Braatz}

National Radio Astronomy Observatory, 520 Edgemont Road, Charlottesville, VA 22903, USA

E-mail: jbraatz@nrao.edu

\begin{abstract}
Narrow-Line Seyfert 1 (NLS1) galaxies are a class of active galactic nuclei (AGN) that have all the properties of type 1 Seyfert galaxies but show peculiar characteristics in the optical (narrowest Balmer lines and strongest FeII emission) and in the X-rays. Recently, we have also discovered that the detection rate of water maser emission in NLS1 is surprisingly high, much higher than typically found in type $1 \mathrm{AGN}$ and similar to that of Seyfert 2 galaxies. In this talk, we shed light on this unexpected result in the framework of the unified model for AGN. In particular, we introduce the case of the recently-discovered INTEGRAL source IGR J16385-2057, classified as a NLS1 galaxy and characterized by an unusual radio continuum and maser emission.
\end{abstract}

The Extreme and Variable High Energy Sky - extremesky2011,

September 19-23, 2011

Chia Laguna (Cagliari), Italy

\footnotetext{
* Speaker.
} 


\section{Introduction}

Water masers associated with AGN typically trace circumnuclear accretion disks (e.g., for UGC 3789: [13]), the inner parts of relativistic jets (e.g., Mrk348; [12]), or nuclear outflows (Circinus; [6]). VLBI studies of $\mathrm{H}_{2} \mathrm{O}$ maser sources, complemented by single-dish monitoring, are unique methods for mapping accretion disks and estimating the enclosed masses ([9]) as well as determining the shock speed and densities of radio jets ([12]). They can also trace the velocity and geometry of nuclear winds, as in the case of Circinus (Greenhill et al. 2003).

So far, more than 3000 galaxies have been searched for water maser emission and detections have been obtained in about 150 of them (Braatz et al., in prep), the majority being Sy 2 galaxies (active galaxies with narrow emission line optical spectra). Maser emission has been detected, instead, only in a handful of type 1 Seyfert galaxies. Interestingly, three galaxies among these, NGC 4051 ([7]), NGC 5506 ([1]), and Mrk 766 ([16]), are classified as Narrow Line Seyfert 1 (NLS1), having all properties of type 1 Seyfert galaxies but with the narrowest Balmer lines from the Broad Line Region and the strongest Fe II emission. NLS1s have also quite extreme X-rays properties, such as a strong soft excess emission below $1 \mathrm{keV}$ and/or rapid flux variability (see, e.g., [8]; [11]). Possible explanations for their peculiar characteristics include very high accretion rates, strong radiation-pressure outflows, and specific viewing angles.

\section{Observations and data reduction}

During March 2010, we searched for water maser emission with the GBT in a sample of 17 Seyfert galaxies discovered by INTEGRAL as sources of hard X-rays between 20 and $40 \mathrm{keV}$ ([2]; Castangia et al. in prep.). In addition, early in 2011, we performed a sensitive GBT search for water maser emission from a sample of 51 NLS1s.

We have also retrieved VLA observations of the water maser line in NGC 4051 and NGC 5506 available from the NRAO archive. We complemented these observations with archival broad-band VLA A-array 22-GHz continuum data of NGC 5506.

The GBT data were reduced and analyzed using the GBTIDL software package. The VLA data were treated through standard tasks implemented in AIPS.

\section{Results and future work}

In our surveys, we obtained two new detections: one in IGR J16385-2057 (hereafter IGR 16385) and one in IRAS $03450+0055$. These two new detections bring the number of NLS1 galaxies showing water emission to five: NGC 4051, NGC 5506, Mrk 766, IGR 16385, and IRAS 03450+0055.

By collecting all (to our knowledge) past NLS1 galaxies searched in the 22-GHz water maser line, we compute an overall water maser detection rate in NLS1 galaxies of $\sim 7 \%$ for the full sample and a detection rate of $\sim 21 \%$ for a volume-limited $(\mathrm{V}<10000 \mathrm{~km} / \mathrm{s})$ sample, demonstrating a connection between NLS1s and the water maser phenomenon ([16]).

Our interferometric study with the VLA on NGC 4051 and NGC 5506 indicates that, for both galaxies, the maser features are associated, with subarcsecond accuracy, with the compact radio 
continuum knots detected in the nuclear region (for NGC 4051: [5]; for NGC 5506: [10]), suggesting that the water maser emission may arise from a molecular disk or from a nuclear jet/wind.

In addition, among the five NLS1s hosting water maser emission, the galaxy IGR 16385 has attracted particular attention due to its peculiar nature. Indeed, IGR 16385 has an elliptical morphology in the optical and the NVSS map shows a $7 \mathrm{mJy}$ radio core coincident with the optical position of the galaxy, quasi-symmetrically surrounded by two extended lobes of emission (Fig. 1). By using a 325-MHz map taken from the 'Westerbork In the Southern Hemisphere' (WISH) archive, we estimated a spectral index for the nuclear region and the lobes typical for optically thin synchrotron emission. The double-lobed structure, although its association with IGR 16385 still needs to be confidently assessed, resembles that of classical radio galaxies. In particular, both the core-to-total power ratio and the source extension are consistent with known radio galaxies of comparable luminosity (e.g., [4]). While sensitive radio continuum maps with higher spatial resolution are necessary to better locate the radio nucleus and characterize the nuclear region (presence of radio jets and flat radio spectrum components), IGR 16385 may constitute one of the rare cases of radio galaxies hosting maser emission (see, e.g., [3] for NGC 1052; [14], [15] for 3C 403).

In order to determine the origin of the maser emission and deriving kinematic information in IGR 16385, as well as in the other four masing NLS1 galaxies, spectral line, high angular resolution (Very Long Baseline Interferometry; VLBI) observations are needed. Furthermore, an extensive comparative study of the main characteristics of masing and non-masing NLS1s is advisable.

\section{Acknowledgments}

A.T. would like to thank Matteo Murgia for useful discussion.

\section{References}

[1] J. A. Braatz et al.: The discovery of five new $\mathrm{H}_{2} \mathrm{O}$ megamasers in active galaxies, ApJ 437 (1994) L99

[2] P. Castangia et al.: $\mathrm{H}_{2} \mathrm{O}$ maser and hard $\mathrm{X}$-ray emission in AGN. In: Proceedings of the Meeting The Extreme and Variable High Energy Sky, PoS (Extremesky 2011) 068

[3] M. J. Claussen et al.: The Water Masers in the Elliptical Galaxy NGC 1052, ApJ 500 (1998) L129

[4] G. Giovannini et al.: Radio nuclei in elliptical galaxies, A\&A 199 (1988) 73

[5] M. Giroletti \& F. Panessa: The Faintest Seyfert Radio Cores Revealed by VLBI, ApJ 706 (2009) 260

[6] L. J. Greenhill et al.: A Warped Accretion Disk and Wide-Angle Outflow in the Inner Parsec of the Circinus Galaxy, ApJ 590 (2003) 162

[7] Hagiwara et al.: A search for extragalactic $\mathrm{H}_{2} \mathrm{O}$ maser emission towards IRAS galaxies - II. Discovery of an $\mathrm{H}_{2} \mathrm{O}$ maser in the type 1 Seyfert galaxy NGC 4051, MNRAS 344 (2003) L53

[8] S. Komossa et al.: Narrow-line Seyfert 1 Galaxies, RMxAC 32 (2008) 86

[9] C. Y. Kuo et al.: The Megamaser Cosmology Project. III. Accurate Masses of Seven Supermassive Black Holes in Active Galaxies with Circumnuclear Megamaser Disks, ApJ 727 (2011) 20

[10] E. Middelberg et al.: Motion and properties of nuclear radio components in Seyfert galaxies seen with VLBI, A\&A 417 (2004) 925 


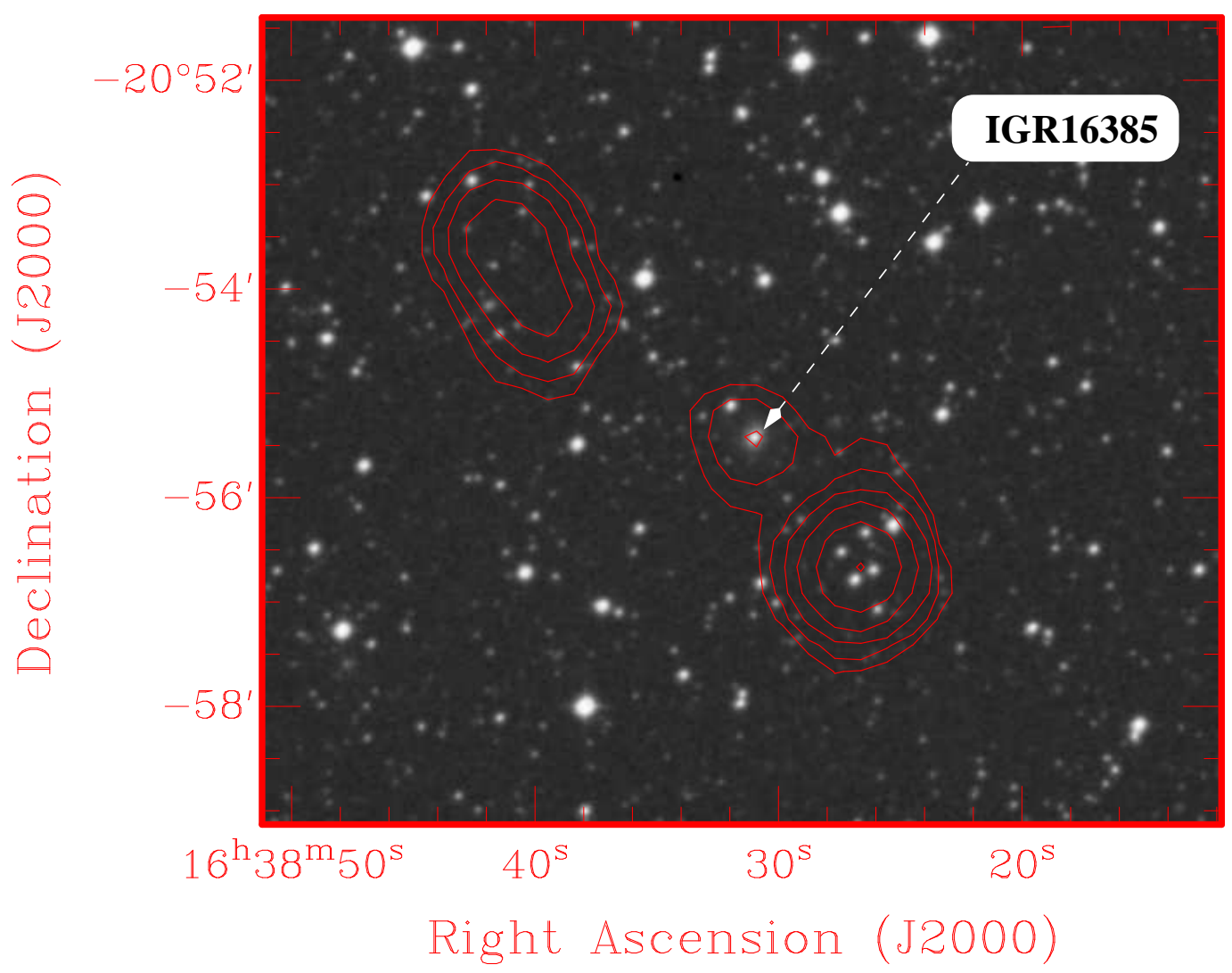

Figure 1: NVSS radio continuum map at $1.4 \mathrm{GHz}$ of IGR 16385 overimposed onto a DSS optical image. Contours are $1.5 \times-1,1,2,4,8,16,32 \mathrm{mJy}$. The white arrow marks the location of the optical galaxy. A $7 \mathrm{mJy}$ radio core is visible, coincident with the optical position of the galaxy and quasi-symmetrically surrounded by two extended lobes of emission. The double-lobes structure resembles that of classical radio galaxy.

[11] F. Panessa et al.: Narrow-line Seyfert 1 galaxies at hard X-rays, MNRAS 417 (2011) 2426

[12] A. B. Peck et al.: The Flaring $\mathrm{H}_{2} \mathrm{O}$ Megamaser and Compact Radio Source in Markarian 348, , ApJ 590 (2003) 149

[13] M. J. Reid et al.: The Megamaser Cosmology Project. I. Very Long Baseline Interferometric Observations of UGC 3789, ApJ 695 (2009) 287

[14] A. Tarchi et al.: Discovery of a luminous water megamaser in the FRII radiogalaxy 3C 403, A\&A 407 (2003) L33

[15] A. Tarchi et al.: The innermost region of the water megamaser radio galaxy 3C 403, A\&A 475 (2007) 497

[16] A. Tarchi et al.: Narrow-line Seyfert 1 galaxies: an amasing class of AGN, A\&A 532 (2011) 125 